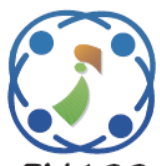

\title{
Compact Dual-Polarized Eight-Element Antenna with High Isolation for 5 G Mobile Terminal Applications
}

\author{
Qasim Hadi Kareem ${ }^{1,2 *}$ \\ Malik Jasim Farhan ${ }^{1}$ \\ ${ }^{1}$ Electrical Engineering Department, Mustansiriyah University, Baghdad, Iraq \\ ${ }^{2}$ Computer Engineering Department, Al-Farabi University College, Baghdad, Iraq \\ * Corresponding author's Email: qasim.hadi2017@ gmail.com
}

\begin{abstract}
This paper proposes a dual-polarized compact eight-element antenna system with high isolation for $5 \mathrm{G}$ mobile terminal applications, consisting of four elements symmetrically located on the system plane's long edges, while the other four are symmetrically placed on both sides of the substrate surface (upper and lower). The proposed antenna system can, therefore, produce a dual-polarized pattern that can operate with a wide bandwidth (3200-3900 $\mathrm{MHz}$ ) and therefore cover Long Term Evolution (LTE) 42 (3400-3600 MHz) band and LTE 43 (3600-3800 MHz) band. The equivalent circuit model for the proposed design is analyzed using Advanced System Design (ADS) tool, and the behaviour of the circuit is compared to the Computer Simulation Technology (CST) tool results and shows a match. The designed system displays a reasonable 10-dB effective impedance bandwidth, high isolation levels above 15-dB, lower Envelope Correlation Coefficients (ECC) below 0.01, and an acceptable radiant output. The nominal Mean Effective Gain (MEG) ratio is less than $1 \mathrm{~dB}$ and the maximum channel capacity of 8 x 8 Multi-Input-MultiOutput (MIMO) is $42.3 \mathrm{bps} / \mathrm{Hz}$, with the total operating band being 20-dB Signal to Noise Ratio (SNR). The calculations, analysis, and discussions of the main parameters that characterize the proposed design were verified and show the appropriate matching impedance and high isolation (high performance) between simulated and measured results for the entire operating band.
\end{abstract}

Keywords: Compact antenna, Dual polarization, MIMO antenna, 5G, LTE 42/43 band.

\section{Introduction}

With the rapid evolution of wireless communication systems, the high performance of portable handheld applications with very high data rates is increasingly needed. Although $4 \mathrm{G}$ communication technology is undergoing extensive studies to provide high data rates for the long-term evolution (LTE), the next-generation communication $(5 \mathrm{G})$ is now attracting greater interest and attention [1]. The new $5 \mathrm{G}$ spectrum consists of two sub- $6 \mathrm{GHz}$ bands and a millimeter wave band. The main challenge for smartphones is how to accommodate high-performance $5 \mathrm{G}$ antennas, without affecting the original $4 \mathrm{G}$ antennas [2]. Since the universal $5 \mathrm{G}$ communication standard has not yet been finalized, a massive MIMO system has become the core $5 \mathrm{G}$ technology [3]. Although the integration of a massive MIMO system on a smartphone can increase channel capacity, it also contributes to degraded isolation and efficiency by placing large numbers of antennas in limited space (delivered by a smartphone). The deployment of multiple antennas on a $5 \mathrm{G}$ smartphone is also a challenge for antenna designers to set up antennas in small areas while reducing system integration problems.

To meet the requirement of a closely packed radiators arrangement and optimize the isolation, antenna miniaturization and decoupling techniques have become an underlying need for future antenna designs, which is now a challenging topic for antenna engineers. Moreover, other requirements for antenna design, including low-profile design, easy manufacturing, and high isolation, must be considered to construct an acceptable MIMO antennas array for smartphones [4]. Several 5G MIMO antennas for mobile terminals working at the sub-6 GHz spectrum have been proposed in recent 
years [5-9]. Among these reported 5G MIMO antenna designs, most are -6-dB impedance bandwidth antenna types. For example, a promising 10 -antenna array (or $10 \times 10$ array) for a $5 \mathrm{G}$ multiband MIMO has been reported in [5], and it has a dual-band operation that can cover the LTE bands 42/43 and LTE band 46. However, its ground clearance regions (sized at $150 \times 80 \mathrm{~mm}^{2}$ ) are too large for practical applications in modern smartphones with large screens and narrow frames. Furthermore, its multi-band operation cannot cover the required -10-dB impedance bandwidth LTE band 42 (3400-3600 MHz) and LTE band 43 (3600-3800 $\mathrm{MHz}$ ) for the future sub-6 GHz spectrum. A low profile planner four-antenna with polarization diversity is proposed in [6] that cover LTE 1800/1900 frequency standards for $4 \mathrm{G}$ applications. This type of MIMO covers lower bands, and it does not accommodate the addition of other radiators. In [7] 5 G multi-band MIMO, a successful 8-antenna array has been proposed, with a multi-band network covering GSM1900, LTE 2300(2.3-2.4 GHz), and LTE 2500(2.54-2.62 GHz). Nevertheless, its surface clearance areas are too wide for use in modern mobile applications with large screens and small frames. Therefore, the multi-band operation of LTE 42 (3.4$3.6 \mathrm{GHz})$ and LTE $43(3.6-3.8 \mathrm{GHz})$ for the potential sub- $6 \mathrm{GHz}$ range cannot cover the required service. In [8], the design of the future smartphone application with a bandwidth impedance of $3.4-3.8 \mathrm{GHz}$ was suggested to cover a single side but not both of a mobile PCB, as these are patch antennas. A compact eight-radiator triple-band system is proposed in [9], covering both licensed and unlicensed bands. Good bandwidth and isolation performance are achieved at the expense of large device sizes.

An increased number of antenna elements will optimize channel capacity and other approaches are also available to increase channel capacity through, for example, the use of diversity techniques and pattern diversity [10]. There are different types of antenna diversity such as polarization, space, and diversity of patterns. To produce good results, we can use any diversity scheme or the combination of such diversity schemes. In spatial diversity, diversity is gained by spatially separating the antenna elements [11]. But the scheme requires much more space than other schemes and space is much less available for small devices so that we are unable to position more than four antenna elements. Dual polarization can be a smart idea to add more antennas in a narrow space. Many works have now been done on dual-polarized base stations [12,13] and [10,14], good performance is achieved, but there is little discussion of polarization and smartphone pattern diversity for either 8-port or mid-bands sub-6 GHz.

In this paper, an orthogonal-polarized hybrid eight-element system is proposed for 5 G MIMO mobile terminals based on PIFAs that are prevalent for mobile devices, particularly small devices with a simple configuration operating in the $3.65 \mathrm{GHz}(3.2-$ $3.9 \mathrm{GHz}$ ) band. Eight of the antenna elements are here modified, for the future range of sub- $6 \mathrm{GHz}$, by inverted-F antennas (IFA) covering LTE 42 (3400$3600 \mathrm{MHz})$ band and LTE 43 (3600-3800 MHz) band. Four antennas are symmetrically on the long edges of the system plane, while the other four are placed symmetrically on both sides of the substrate surface (upper and lower). The proposed antenna system can, therefore, produce orthogonal polarization characteristics with the connected ground plane of eight-radiators to enhance the channel capacity by reducing the correlation coefficient and increasing the isolation between multi-antennas. Here, the four modified IFA antennas produce linear polarization (LP) on the ground's long edges while the other four IFAs do radiate the LP wave but on the upper and lower sides of the planner. Increased couple of antenna units will, therefore, induce orthogonal polarization, which will help to increase the efficiency of the MIMO range of eight antennas. In addition, through the analysis, calculation and discussion of the eight-element antenna array, the correlation coefficients (return loss), and the channel capacity show the appropriate matching impedance and good isolation results for the entire operating band.

The following matters are presented in this paper: Section 2 defines the geometry of the dual-polarized system, while section 3 shows results of the proposed antenna in terms of S-parameters, MIMO system performance, efficiency, radiation pattern, and a comparison with the referenced antennas. The conclusion is finally stated in Section 4.

\section{The geometry of the dual-polarized system}

Fig. 1 shows the proposed modified inverted-FA dual-polarized eight-element array for $5 \mathrm{G}$ mobile terminal applications. As shown, the antenna components are mounted on a $60 \times 120 \mathrm{~mm}^{2}$ ground plane and printed on a $1.6 \mathrm{~mm}$ FR4-substrate with 4.3 relative permittivity and tangent loss of 0.026 .

Four antennae components (ant1-ant4) are placed on the two long edges of the ground plane and on the upper and lower side of the system are loaded perpendicularly the other four antennas (ant5-ant8). 


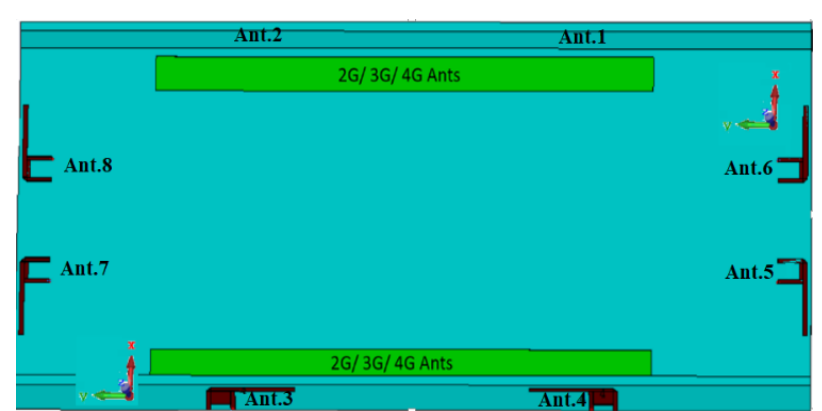

(a)

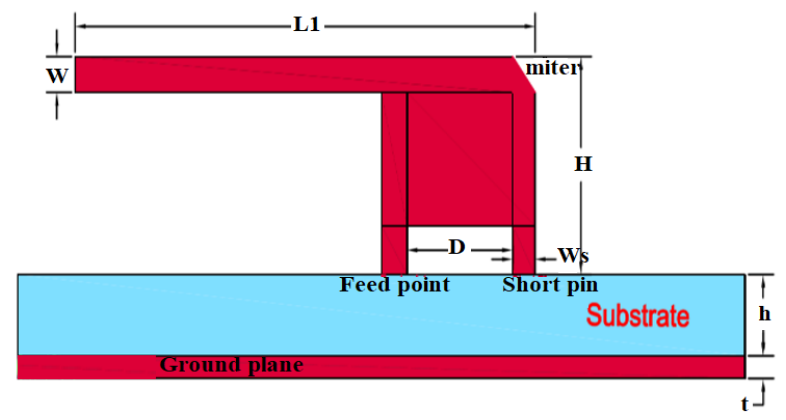

(b)

Figure. 1 Geometry of $8 \times 8$ MIMO antenna design: (a) Dual-polarized eight-element and (b) Single-element

The two rectangular clearance areas marked in green on both sides of the smartphone as shown are reserved for the accommodation of two $2 \mathrm{G} / 3 \mathrm{G} / 4 \mathrm{G}$ wireless network antennas.

\subsection{Geometry of modified inverted-FA}

In the modified inverted-FA (Fig. 1 (b)) the resonant frequency is defined by the length of the patch $\left(L_{1}=12.66\right)$, the width of the patch $(\mathrm{W}=0.67)$, the width of the shorting pin $\left(W_{s}=0.67\right)$, the height of the layer $(\mathrm{h}=1.6)$ and the height of the shorter pin $(\mathrm{H}=3.55)$. The concept is a quarter wavelength of the shorting pin from the opposite edge of the board.

The feed point is on the same side, as seen, and the feed is $\mathrm{D}=2.95$ from the short pin. The IFA is from the ground plane at a height of $\mathrm{H}$ and is on top of a dielectric with 4.3 permittivity. The IFA's impedance can be regulated via the feed distance to the short pin (D). The closer the feed is to the shortening point, the impedance would decrease. By moving it away from the short edge, the impedance can be increased to allow it to be tuned for that parameter. The miter with $45^{0}$ is used to prevent the micro-bending of the right angle, resulting in a low flow of the current on the stub.

The design equation is [15]

$$
L_{1}+W-W s=\frac{\lambda}{4}+h+H
$$

Where $\lambda$ is the wavelength of the dielectric. Eq. (1) reduces to the two special cases which follow

$$
\begin{aligned}
& L_{1}=\frac{\lambda}{4}+h+H \quad \text { for } W-W s \\
& L_{1} \approx-W+\frac{\lambda}{4}+h+H \quad \text { for } W s \approx 0
\end{aligned}
$$

Eq. (1) can be resolved by resonant frequency

$$
f=\frac{c}{4\left(L_{1}+W-W s-h-H\right) \sqrt{\varepsilon_{r}}}
$$

Where $\varepsilon_{r}$ is the substrate's relative permittivity and $\mathrm{c}$ is the free space light speed.

\subsection{Defected ground plane}

An important part of the function of the invertedFA is the ground plane, where the input excitation of currents contributes to the excitation of ground currents. The ground plane's response behaves as a perfect reflector only when it is infinite or much larger than the radiator itself. The required length is generally $\lambda / 4$ of the operating wavelength of the inverted-FA ground plane. If its length is longer than $\lambda / 4$, more side lobes will be shown in the inverted-FA radiation pattern. However, if it is considerably shorter than $\lambda / 4$, it is difficult to tune the antenna and

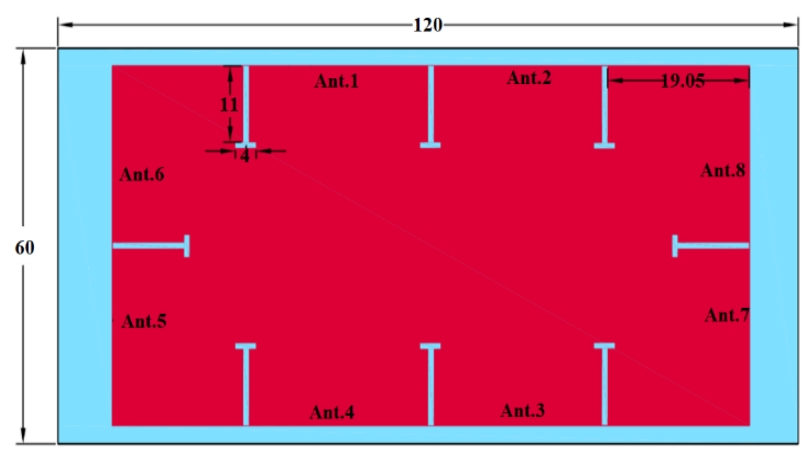

Figure. 2 The defected ground plane of the eight-antenna array

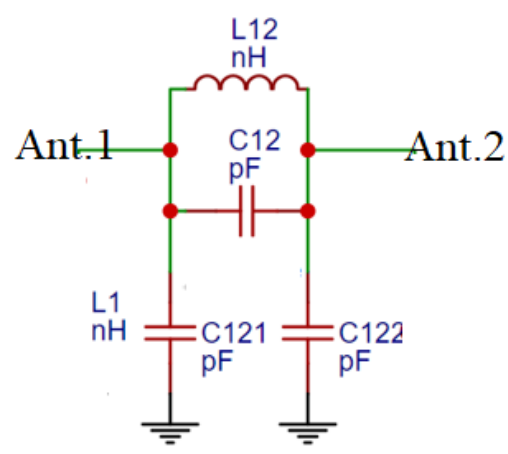

Figure. 3 Isolation circuit between Ant1 and Ant.2 
hence the overall output degrades [15-16]. Fig. 2 shows the defected ground plane which has an approximate value of $\lambda / 4$ and has been removed from below the antenna in order to achieve the good performance of the proposed system in terms of radiation pattern, bandwidth and high isolation between antennas as seen in results of the discussion. The equivalent circuit of the defected ground between Ant. 1 and Ant.2 is shown in Fig. 3. From CST tool, its values are extracted using extraction approach and then assigned in the ADS tool.

\subsection{Equivalent circuit of inverted-F antenna}

To achieve effective radiation, antennas must be a good radiating structure, which means that currents (electrical fields) are added in phase, and the structure has to be able to get the power down the transmission line and therefore to the antenna [15]. To accomplish this, it is desirable to have the reactive component of the impedance to be zero. Fig. 1(b) shows a shorted strip line that is a small fraction of a wavelength creates a parallel inductance to the antenna impedance. Similarly, the open circuit of antenna trace creates a capacitance to the left of the feed. The feed location is chosen to "balance out" the capacitance (to the left of the feed) and the inductance to the right of the feed. The inductance and capacitance cancel out, leaving just the radiation resistance.

The equivalent circuit model shown in Fig. 4 is used as a method for tuning the operation MIMO system, which is completed using an advanced design simulation (ADS) tool. Two different platforms of design microwave components are the CST and ADS. Therefore, the value to be assigned to each circuit element must be extracted from CST and then applied to ADS. In ADS, re-optimization of these circuit components is needed for accurate results.

The circuit and the CST simulated response are compared, as shown in Fig. 5. The ADS' circuit response is shifted towards a lower frequency side,

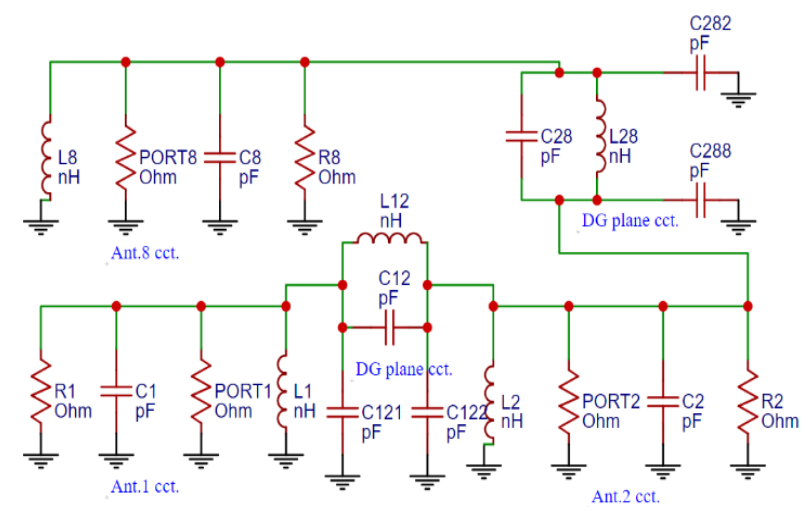

Figure. 4 Equivalent circuit of three inverted-F antennas

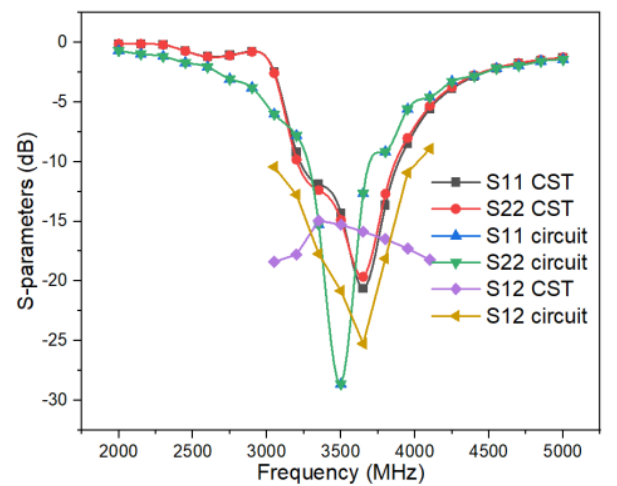

Figure. 5 Circuit response using ADS and CST

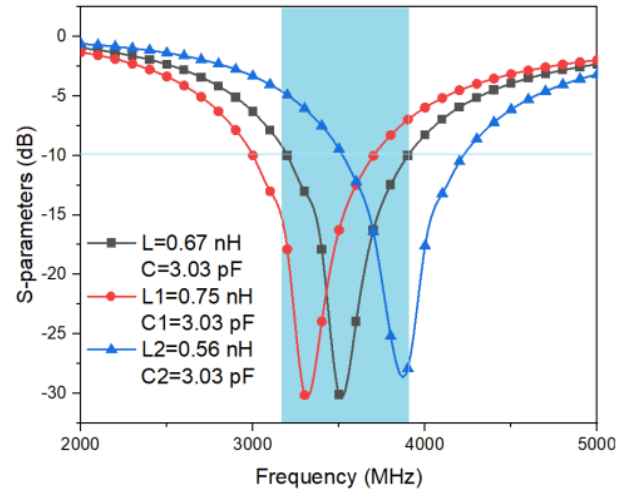

(a)

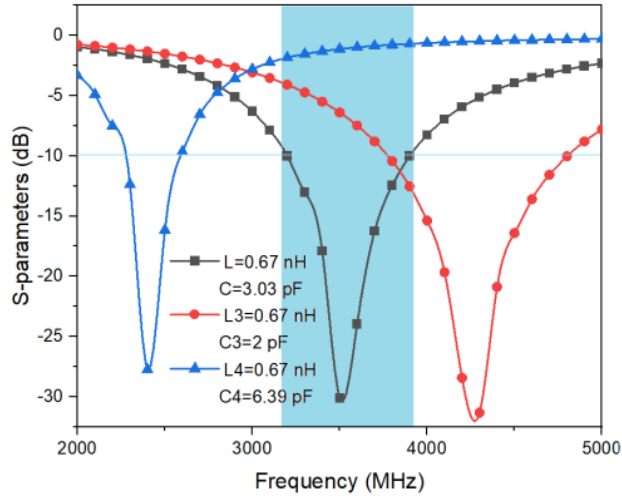

(b)

Figure. 6 Response of equivalent circuit for different values of $\mathrm{L}$ and $\mathrm{C}$ : (a) Constant $\mathrm{C}$ and (b) Constant $\mathrm{L}$

and there is a high isolation parameter than the CST simulated response. The interested frequency band of Ant. 1 is represented by L1-C1-R1 and its defected ground (isolation parameter) by L12-C12-C121C122. The values of circuit parameters are $\mathrm{L} 1=\mathrm{L} 2=\mathrm{L} 8=0.67 \mathrm{nH}, \mathrm{C} 1=\mathrm{C} 2=\mathrm{C} 8=3.03 \mathrm{pF}, \mathrm{L} 12$ $=\mathrm{L} 28=1.41 \mathrm{nH}, \mathrm{C} 12=\mathrm{C} 28=1.3 \mathrm{pF}, \mathrm{C} 121=0.156 \mathrm{pF}$, $\mathrm{C} 122=0.156 \mathrm{pF}$, and $\mathrm{R} 1=\mathrm{R} 2=\mathrm{R} 8=50 \Omega$. Each port is represented by $50 \mathrm{Ohm}$. Exact CST equivalent values are significant to obtain with the ADS.

It is clear from Fig. 6 that when the change in the inductance value causes the displacement of the curve to high or low frequencies at the same bandwidth, while when the capacitance changes, it 
causes the reduce or increase of the bandwidth and also a change in the value of the frequencies.

\section{Results of proposed design}

\subsection{S-parameters}

The simulated S-parameters for both vertical and horizontal antennas is shown in Fig. 7 and the CST Studio V.19 is used as a simulation tool. The resonant frequency of approximately $3650 \mathrm{MHz}$, as shown in Fig. 7(a), covers the operating range and the estimated 10-dB effective impedance range (2:1 VSWR) is $3200-3900 \mathrm{MHz}$, with a resonant mode isolation greater than $15-\mathrm{dB}$.

Although the HAs resonant frequency is moved to the higher frequency (Fig. 7(b)), the desired operating bandwidth is still covered completely. It can thus be concluded that the induced orthogonal polarization has contributed significantly to MIMO's overall performance.

The proposed compact eight-antenna array was manufactured for both views (front and back) as shown in Fig. 8.

The determined S-parameter output is performed by the KC901V network vector analyzer. As pointed out in Fig. 9, the antenna provides very good impedance matching around the band, and high isolation with more than $-17 \mathrm{~dB}$ of mutual coupling

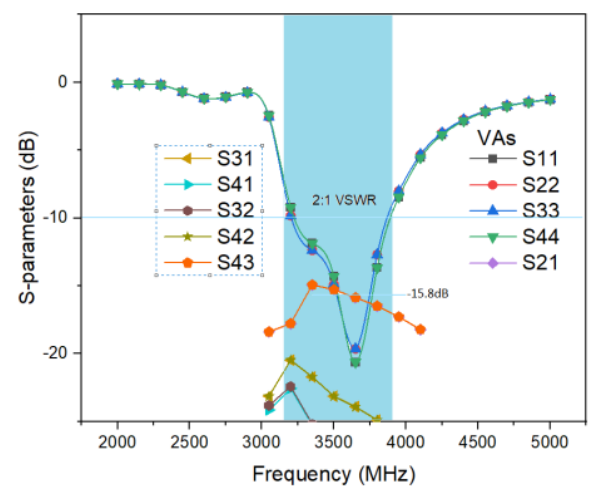

(a)

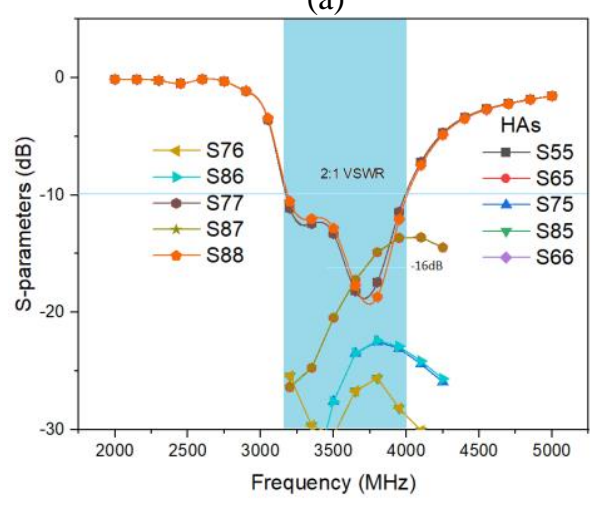

(b)

Figure. 7 Input reflection and transmission coefficients: (a) VAs and (b) Has was obtained for the manufactured sample. The return loss $10 \mathrm{~dB}$ (S33, S55, and S77) bandwidth was approximately (3410-3910) MHz and due to minor manufacturing errors slightly narrower than the simulated ones (3200-3900 MHz). However, for LTE $42 / 43$ bands with a reasonable impedance matching of $10 \mathrm{~dB}$, the antenna can still cover the built-in operating band.

\subsection{MIMO antenna system performance}

Evaluation of the potential MIMO performance of the antenna system is based on ECC, mean effective gain (MEG), channel capacity, and channel capacity loss (CCL). The ECC is usually used to evaluate the multi-antenna system's diversity, where the low ECC value means higher isolation and greater diversity gain. The ECC value should usually be below 0.5 , so that it is possible to achieve good diversity characteristics for mobile terminal applications [17].

Since the detailed formula of ECC for diversity antenna, for brevity, has already been discussed in [18], it is not mentioned. The ECC function determined by the scattering parameters is shown in Fig. 10(a). Its values for the entire operating band are less than 0.01 , which satisfies an appropriate ECC $(<$ $0.5)$ requirement for MIMO operations.

The MEG ratios $\left(\left|M E G_{i} / M E G_{j}\right|<3 d B\right)$ are calculated to evaluate the imbalanced rates of the various pathways of propagation in order to quantify the mean received signal intensity for each radiator [19]. The antenna MEG can be calculated by the 2D gain pattern provided by [20]. As can be seen from Table 1, the MEG for VAs (Ant.1-Ant.4) is varied from -7.39 to $-7.067-\mathrm{dBi}$, and the HAs are varied from -7.076 to $-6.909-\mathrm{dBi}$. Consequently, the MEG variations over the entire operating bandwidth are around 1-dB lower, which is much smaller than the accepted standard $\left(\left|M E G_{i} / M E G_{j}\right|<3 d B\right)$. A good balance of power and a low loss of diversity can, therefore, be ensured.

The channel capacity in Fig. 10 (b) is accomplished by providing the same power to be assigned to each antenna at transmitter and Rayleigh channel is the propagation scenario, with $20 \mathrm{~dB}$ SNR at the end of the receiver and an average fading of over 1000 Rayleigh in the dual-polarized antenna system [21, 22].

The capacity of the proposed $8 \times 8$ MIMO system is $42.3 \mathrm{bps} / \mathrm{Hz}$ through the operating band, and the channel capacity reached $46 \mathrm{bps} / \mathrm{Hz}$ for an ideal 8 x 8 MIMO antenna. The expected achievable data throughput can reach a speed of up to 30.1-Gbps (20- 
Table 1. Calculated MEG from two-dimensional gain

\begin{tabular}{ccccccccc}
\hline $\begin{array}{c}\text { Freq. } \\
{[\mathrm{GHz}]}\end{array}$ & MEG 1 & MEG 2 & MEG 3 & MEG 4 & MEG 5 & MEG 6 & MEG 7 & MEG 8 \\
\hline 3.4 & -7.392 & -7.392 & -7.396 & -7.396 & -6.909 & -6.928 & -6.909 & -6.928 \\
3.6 & -7.185 & -7.186 & -7.184 & -7.183 & -7.066 & -7.076 & -7.065 & -7.075 \\
3.8 & -7.070 & -7.071 & -7.068 & -7.067 & -6.927 & -6.908 & -6.926 & -6.906 \\
\hline
\end{tabular}

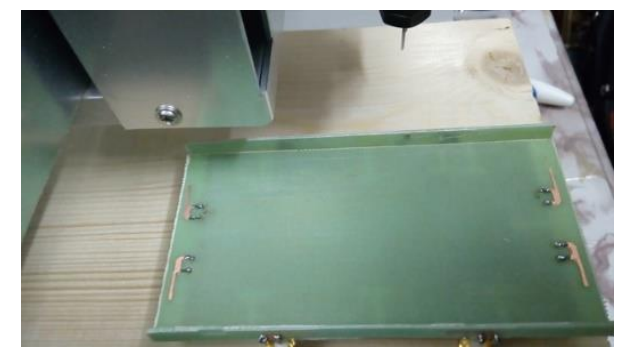

(a)

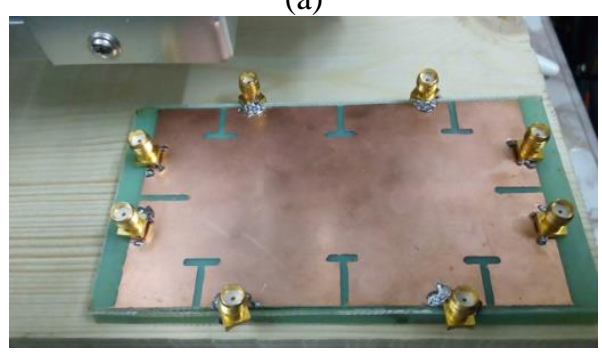

(b)

Figure. 8 Photos of the fabricated 5G MIMO antenna System: (a) front and side view and (b)back view

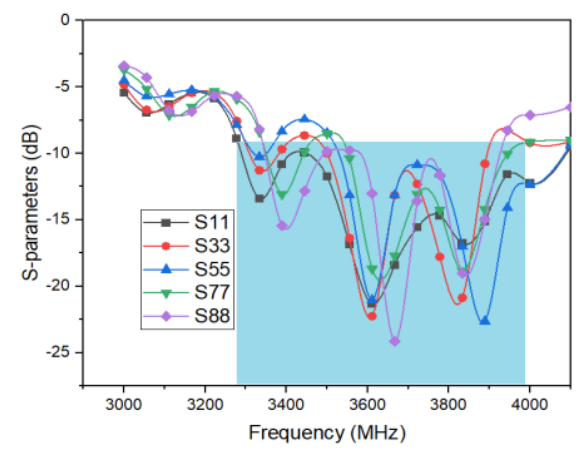

(a)

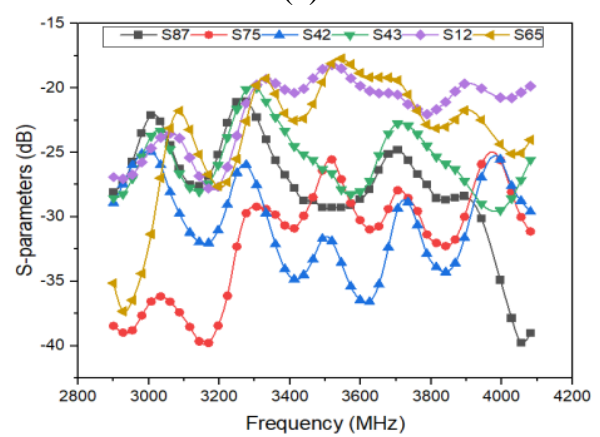

(b)

Figure. 9 Measured S-parameters for both VAs and Has: (a) Input reflection and (b) Transmission coefficients

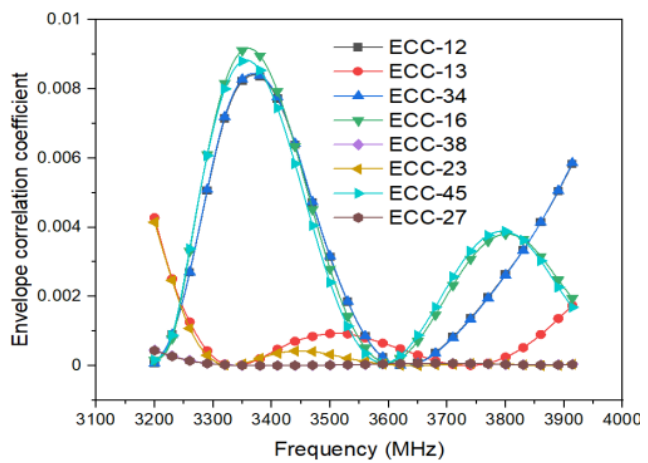

(a)

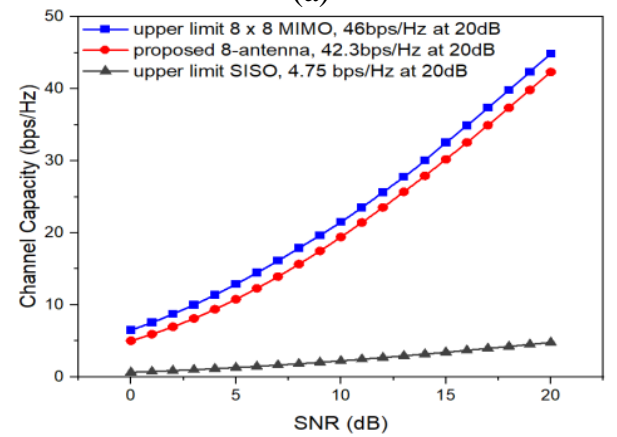

(b)

Figure. 10 (a) Envelop correlation coefficient and (b) Ergodic channel capacities

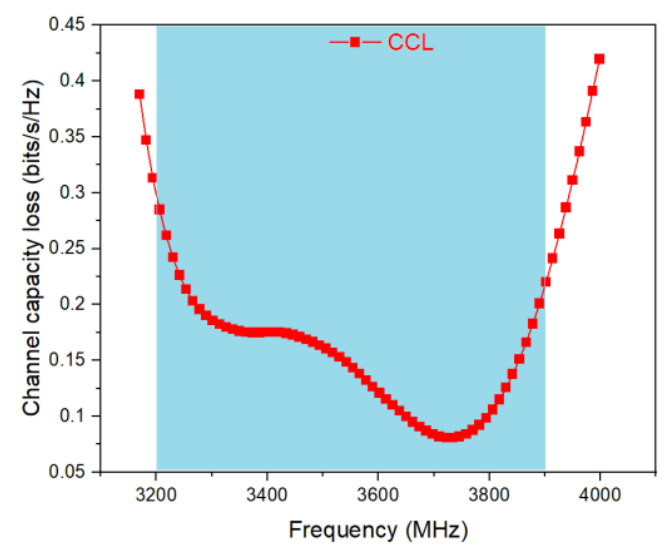

Figure. 11 Channel capacity loss of the proposed MIMO antenna

dB SNR) within the $3.2-3.9 \mathrm{GHz}$ band when multiplied by the absolute bandwidth $(700 \mathrm{MHz})$. The orthogonal polarization technique, therefore, showed a good channel capacity close to the ideal 
case. (46 bps/Hz) and 8.9 times the conventional SISO system as shown in Fig. 10 (b).

In theory, by increasing the number of MIMO elements, the channel capacity could be increased. The uncorrelated Rayleigh deterioration however leads to the MIMO system's channel capacity loss (CCL). The loss of capacity $\left(C_{\text {loss }}\right)$ for the antenna system can be computed by using the following equation $[23,24]$ :

$$
C_{\text {loss }}=-\log _{2} \operatorname{det}\left(\psi^{R}\right)
$$

Where $\psi^{R}$ is the matrix of correlation of the receiving antenna given by

$$
\begin{gathered}
\psi^{R}=\left[\begin{array}{ccc}
\rho_{11} & \cdots & \rho_{18} \\
\vdots & \ddots & \vdots \\
\rho_{81} & \cdots & \rho_{88}
\end{array}\right] \\
\rho_{i i}=1-\left|\sum_{m=1}^{8} S_{i m}^{*} S_{m i}\right|, \text { and } \\
\rho_{i j}=-\left|\sum_{m=1}^{8} S_{i m}^{*} S_{m j}\right|, \text { for } i, j=1, \ldots, 8
\end{gathered}
$$

Fig. 11 shows a simulated loss in the capacity of the designed antenna system, where the simulated losses of capacity in an operating frequency band are less than $0.4 \mathrm{bps} / \mathrm{Hz}$, an acceptable CCL limit [25].

The proposed orthogonal-polarized hybrid eightelement array can, therefore, provide desirable performance in the MIMO system on the basis of the calculated ECC, MEG, CCL, and channel capacity as described above.

\subsection{Efficiency and radiation pattern}

Total efficiency and electric field patterns for the proposed design are considered in order to verify the radiative performance. The total antenna efficiency for various polarizations is shown in Fig. 12, with an approximate 83-96\% (VAs) and 82-96\% (HAs).

As can be seen, the antenna offers high efficiency even though the FR-4 dielectric is $1.6 \mathrm{~mm}$ thick.

Fig. 13 demonstrates the structure of the proposed design in which measurements of the radiation pattern can be made. A directional antenna was used in the field measurement method for transmitting antenna, while the antenna under test (AUT) acts as a receiving antenna. The transmitting antenna is situated at a distance $(\mathrm{R})$ of one meter from the AUT. Two VNA ports with low-loss high frequency RF cables were used to connect the AUT and transmit the antenna. To measure the transmission coefficients, the VNA has interfaced with a personal computer with a LAN cable.

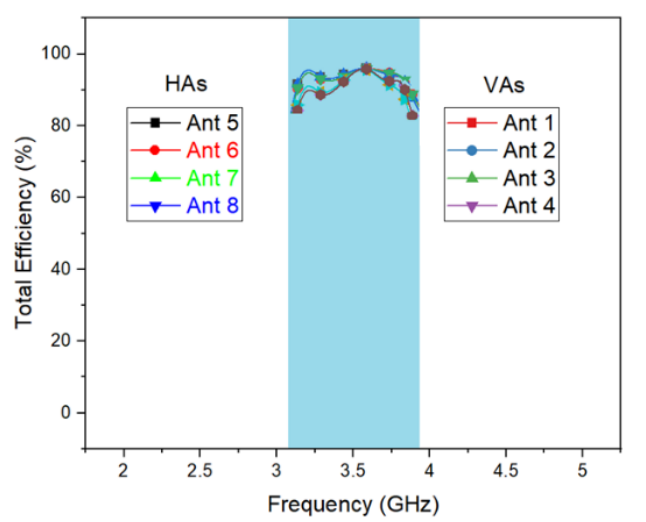

Figure. 12 Total antenna efficiency for dual-polarized

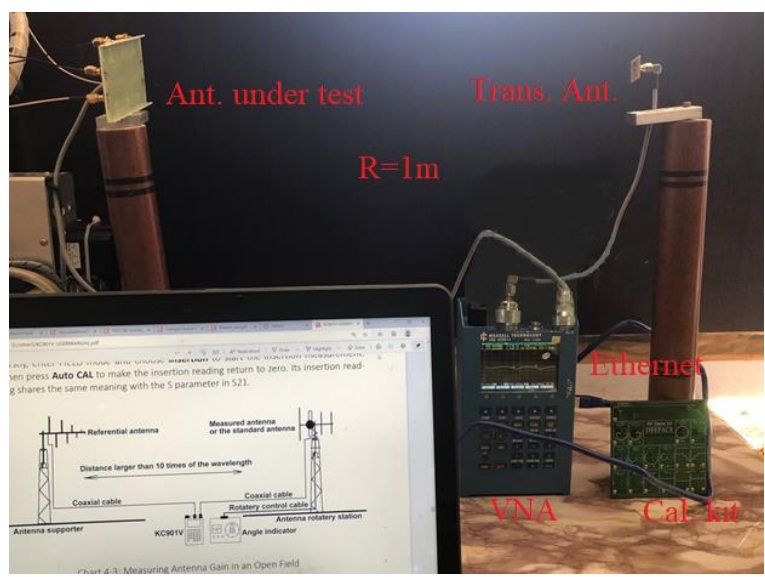

Figure. 13 Complete structure of the fabricated design

Fig. 14 (at $3.65 \mathrm{GHz}$ ) shows the simulated and measurements of E-theta and E-phi radiation patterns for the eight-element in the xy-plane. It is noted that the vertical antennas (Ants.1 - 4) return high radiation at the phi $=21$ (for Ant.1), phi $=201$ (forAnt.2), phi = 339 (forAnt.3), and phi $=159$ (forAnt.4) in the $+x$ direction (forAnt.1, 3) and -x direction (forAnt.2, 4) at $3.65 \mathrm{GHz}$. For the horizontal antennas (Ants.5- 8), at $3.65 \mathrm{GHz}$, high radiation is typically phi-oriented at the phi $=236$ (for Ant.5), phi $=304$ (for Ant.6), phi $=125$ (for Ant.7), and phi = 56 (for Ant.8) in the $-\mathrm{y}$ direction (for Ant.5, 6) and +y direction (for Ant.7, $8)$. Here, it can be seen that horizontal and vertical antennas have clearly complementary and symmetrical patterns which guarantee good pattern diversity and a good linear polarization purity. The co-polarizing component (E-phi) is 13.7-13.75-dB (for Ants.1-4) and 20.11-20.2-dB (for Ants.5-8) greater than its corresponding cross-polarizing component (E-theta) in the direction of the main lobe. The diversity in terms of polarization and pattern is thus achieved for the current designed system that benefits the MIMO utility. 


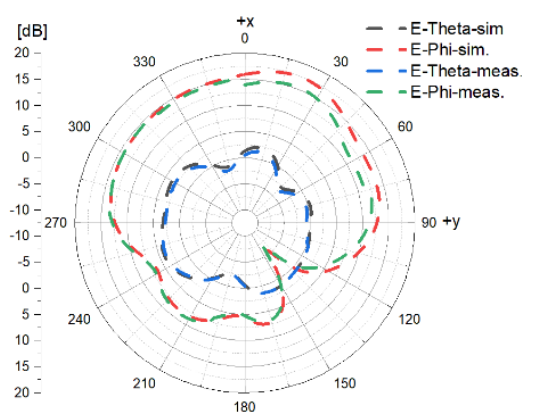

(a)

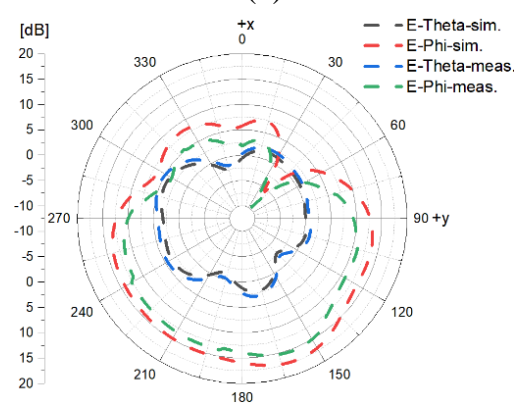

(d)

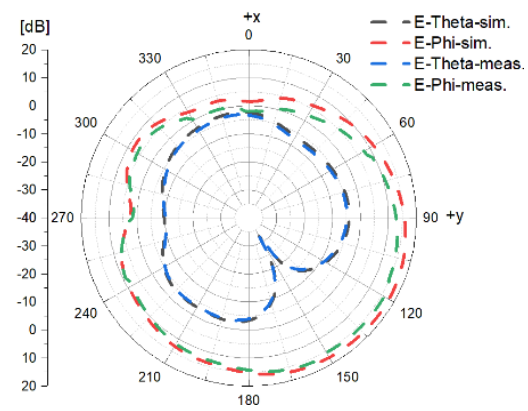

(g)

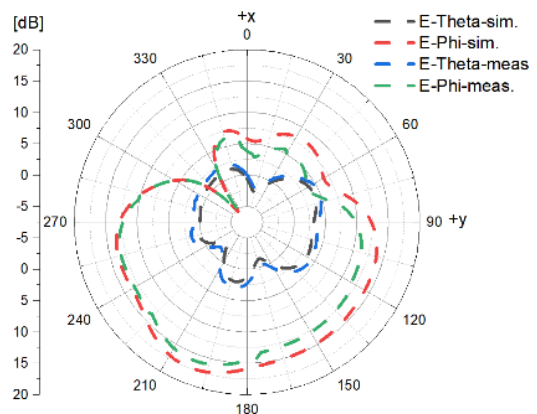

(b)

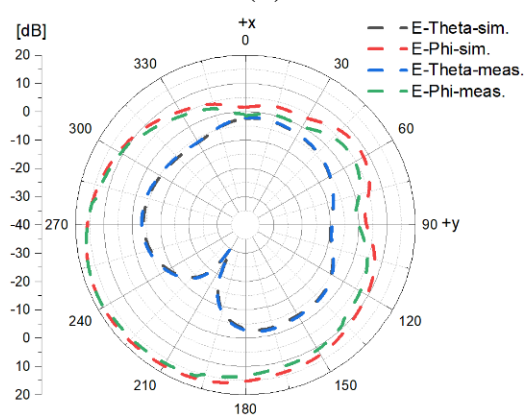

(e)

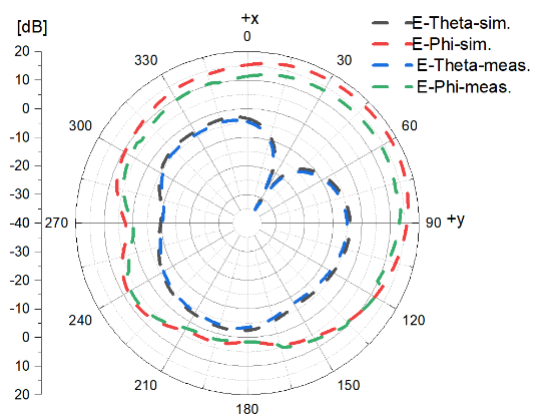

(h)

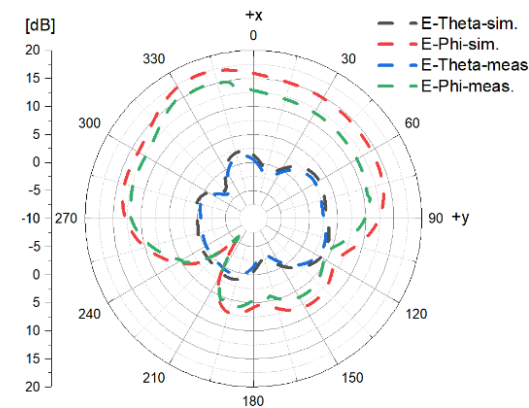

(c)

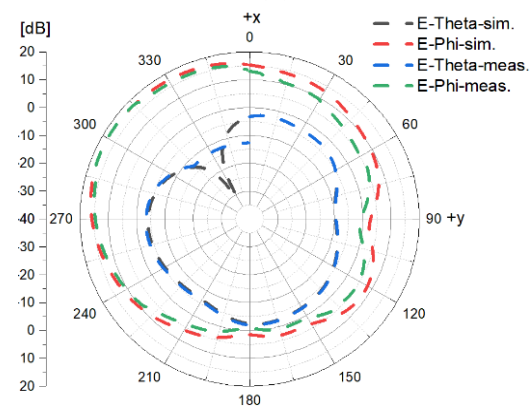

(f)

Figure. 14 Radiation patterns of the proposed MIMO antenna system in the xy plane: (a) Ant1, (b) Ant2, (c) Ant3, (d) Ant4, (e) Ant5, (f) Ant6, (g) Ant7, and (h) Ant8

\subsection{Comparison with antenna referenced}

Table 2 compares the proposed work with the references considered. The proposed MIMO antenna has several advantages as compared with the existing antennas reported in the literature. The designed system consists of eight radiators that offer additional freedom to improve link reliability and high channel capacity $(42.3 \mathrm{bps} / \mathrm{Hz})$. A good performance in terms of a broad bandwidth $(700 \mathrm{MHz})$, high efficiency (over $82 \%$ ), and high isolation $(>15 \mathrm{~dB}$ ) are achieved by introducing the defected ground plane, which has an approximate value of $\lambda / 4$ and has been removed from below the radiator without compromising thesize, complexity, and radiation performance of the antenna. The radiating elements are located in both vertical and horizontal planes, which produces additional polarization vectors. The orthogonal alignment of radiating antennas introduces pattern diversity, which helps achieve high isolation between radiators without employing any complex decoupling elements. The total area occupied by the proposed MIMO antenna system structure is relatively small $\left(120 \times 60 \mathrm{~mm}^{2}\right)$ and can be easily packed into a mobile terminal.

\section{Conclusion}

In this paper, a compact eight-port dually polarized hybrid antenna arrays were proposed for future sub-6 $\mathrm{GHz}$ MIMO applications on smartphones operating in 42, $43(3400-3800 \mathrm{MHz})$ LTE bands. The proposed eight-antenna system equivalent circuit model is analyzed using ADS tool, and consists of vertical and horizontal inverted-FA components supported for better isolation between MIMO antennas. The antenna system displays a reasonable 10-dB effective impedance bandwidth, high isolation levels above 15-dB, lower ECCs below 0.01 , and an acceptable radiant output. 
Table 2. Comparison the work in progress with the antennas referenced

\begin{tabular}{|c|c|c|c|c|c|c|c|}
\hline Ref. & BW. (GHz) & $\begin{array}{l}\text { Eff. } \\
(\%)\end{array}$ & $\begin{array}{c}\text { Isolation } \\
(\mathrm{dB})\end{array}$ & $\begin{array}{c}\text { Ant. elem. \& } \\
\text { PCB Size }\left(\boldsymbol{m m}^{3}\right)\end{array}$ & ECC & $\begin{array}{l}\text { Peak CC } \\
\text { (bps/Hz) }\end{array}$ & $\begin{array}{l}\text { No. of } \\
\text { elements }\end{array}$ \\
\hline [5] & $3.4-3.8$ & $42-65$ & $>11$ & $16.2 \times 3 \times 0.8$ & $<0.15$ & 48 & $10 \times 10$ \\
\hline & $\begin{array}{c}5.15-5.925 \\
(-6 \mathrm{~dB})\end{array}$ & $62-82$ & & $150 \times 80 \times 0.8$ & 0.05 & $\begin{array}{c}51.4 \\
\text { 20-dB SNR }\end{array}$ & \\
\hline [6] & $\begin{array}{l}3.4-3.6 \\
(-6 \mathrm{~dB})\end{array}$ & $40-52$ & $>10$ & $\begin{array}{c}10 \times 1 \times 3.1 \\
150 \times 75 \times 0.8\end{array}$ & $<0.15$ & $\begin{array}{c}36 \\
\text { 20-dB SNR }\end{array}$ & $8 \times 8$ \\
\hline [7] & $\begin{array}{l}3.4-3.6 \\
(-6 \mathrm{~dB})\end{array}$ & $59-72$ & Not M. & $5 \times 2 \times 5$ & $\begin{array}{l}\text { Not } \\
\text { M. }\end{array}$ & 15 & $2 \times 8$ \\
\hline [8] & $\begin{array}{l}3.4-3.8 \\
(-6 \mathrm{~dB})\end{array}$ & $40-62$ & $>10$ & $\begin{array}{c}8 \times 3 \times 0.8 \\
140 \times 70 \times 0.8\end{array}$ & $<0.1$ & $\begin{array}{c}47 \\
20-\mathrm{dB} \text { SNR }\end{array}$ & $10 \times 10$ \\
\hline [9] & $3.4-3.8$ & $70-80$ & $>13$ & $\begin{array}{c}25 \times 25 \times 1.6 \\
150 \times 75 \times 1.6\end{array}$ & $<0.01$ & Not M. & $8 \times 8$ \\
\hline [10] & $\begin{array}{l}3.4-3.6 \\
(-10 \mathrm{~dB})\end{array}$ & $\begin{array}{l}43- \\
52.5\end{array}$ & $>12$ & $\begin{array}{c}25 \times 7 \times 1.5 \\
150 \times 75 \times 0.8\end{array}$ & $<0.13$ & Not M. & $8 \times 8$ \\
\hline [11] & $\begin{array}{l}1.68-2.24 \\
(-10 \mathrm{~dB})\end{array}$ & 95.5 & $>13$ & $82.7 \times 82.7$ & $<0.01$ & Not M. & $4 \times 4$ \\
\hline [12] & $\begin{array}{l}3.4-3.6 \\
(-6 \mathrm{~dB})\end{array}$ & $30-53$ & $>12$ & $\begin{array}{c}8 \times 3 \times 0.8 \\
150 \times 75 \times 0.8\end{array}$ & $<0.35$ & $\begin{array}{c}70 \\
\text { 20-dB SNR }\end{array}$ & $16 \times 16$ \\
\hline [14] & $\begin{array}{l}3.4-3.6 \\
(-10 \mathrm{~dB})\end{array}$ & $62-78$ & $>10$ & $\begin{array}{l}16.7 \times 3 \times 0.8 \\
140 \times 70 \times 0.8\end{array}$ & $<0.2$ & $\begin{array}{c}39 \\
\text { 20-dB SNR }\end{array}$ & $8 \times 8$ \\
\hline [22] & $\begin{array}{l}1880-1920 \\
2300-2400 \\
2540-2620\end{array}$ & $\begin{array}{l}40-52 \\
52-70\end{array}$ & $>10$ & $\begin{array}{l}14 \times 15 \times 1 \\
136 \times 68.8 \times 1\end{array}$ & $<0.1$ & Not M. & $8 \times 8$ \\
\hline [26] & $\begin{array}{c}2.55-2.65 \\
(-10 \mathrm{~dB})\end{array}$ & $48-63$ & $>12.5$ & $\begin{array}{l}31.2 \times 5 \times 2 \\
136 \times 68 \times 1\end{array}$ & $<0.15$ & $\begin{array}{c}40 \\
20-\mathrm{dB} \text { SNR }\end{array}$ & $8 \times 8$ \\
\hline Present work & $\begin{array}{l}3.2-3.9 \\
(-10 \mathrm{~dB})\end{array}$ & $82-96$ & $>15$ & $120 \times 60 \times 1.6$ & $<0.01$ & $\begin{array}{c}42.3 \\
\text { 20-dB SNR }\end{array}$ & $8 \times 8$ \\
\hline
\end{tabular}

The nominal MEG ratio is less than $1 \mathrm{~dB}$ and the maximum channel capacity of $8 \times 8$ MIMO is 42.3 bps / Hz, with the total operating band being $20-\mathrm{dB}$ SNR, 8.9- times the conventional SISO antenna system. Because of its good performance and simplicity of structure, the design of the antenna proposed for $5 \mathrm{G}$ MIMO applications on portable devices would be a very good choice. Based on the findings, future work can be done to design and calculate the effect of the handset antennas' radiation pattern on the human head using the CST simulation program.

\section{Conflicts of interest}

The authors declare that there is no conflict of interest.

\section{Author contributions}

Q. Hadi devised the idea, the main conceptual ideas and proof outline. M. Jasim was involved in planning and supervised the work. Q. Hadi processed the experimental data, performed the analysis with CST and ADS software, drafted the manuscript and manufactured the prototype and characterized them with 2-port vector network analyzer. M. Jasim aided in interpreting the results and worked on the manuscript.

\section{Acknowledgments}

The authors would like to thank Mustansiriyah University (www.uomustansiriyha.edu.iq) Baghdad, Iraq for its support in the present work.

\section{References}

[1] J. G. Andrews, S. Buzzi, W. Choi, S. V. Hanly, A. Lozano, A. C. K. Soong, and J. C. Zangh, "What Will 5G Be?", IEEE Journal on Selected Areas in Communications, Vol. 32, No. 6, pp. 1065-1082, 2014.

[2] C. Deng, Y. Li, Z. Zhang, and Z. Feng, "Planar Printed Multi-Resonant Antenna for Octa-Band 
WWAN/LTE Mobile Handset", IEEE Antennas and Wireless Propagation Letters, Vol. 14, pp. 1734-1737, 2015.

[3] G. J. Foschini and M. J. Gans, "On Limits of Wireless Communications in a Fading Environment When Using Multiple Antennas", Wireless Personal Communications, Vol. 6, pp. 311-335, 1998.

[4] H. H. Yang and Y. Q. S. Quek, Massive MIMO meet small cell, Springer, 2017.

[5] Y. Li, C. Sim, Y. Luo, and G. Yang, "Multiband 10-Antenna Array for Sub-6 GHz MIMO Applications in 5-G Smartphones", IEEE Access, Vol. 6, pp. 28041-28053, 2018.

[6] Z. Qin, W. Geyi, M. Zhang, and J. Wang, "Printed eight-element MIMO system for compact and thin 5G mobile handset", Electronics Letters, Vol. 52, pp. 416-418, 2016.

[7] A. A. Hadi, J. Ivonen, R. Valkonen, and V. Viikari, "Eight element antenna array for diversity and MIMO mobile terminal in LTE $3500 \mathrm{MHz}$ band", Microwave Optical Technology Letters, Vol. 56, pp. 1323-1327, 2014.

[8] H. Wang, R. Zhang, Y. Luo, and G. Yang, "Compact Eight-Element Antenna Array for Triple-Band MIMO Operation in 5G Mobile Terminals", IEEE Access, Vol. 8, pp. 1943319449, 2020.

[9] K. Wong, C. Tsai, and J. Lu, "Two Asymmetrically Mirrored Gap-Coupled Loop Antennas as a Compact Building Block for EightAntenna MIMO Array in the Future Smartphone", IEEE Transactions on Antennas and Propagation, Vol. 65, No. 4, pp. 1765-1778, 2017.

[10] M. Li, Y. Ban, Z. Xu, G. Wu, C. Sim, K. Kang, and $\mathrm{Z}$. Yu, "Eight-Port Orthogonally DualPolarized Antenna Array for 5G Smartphone Applications", IEEE Transactions on Antennas and Propagation, Vol. 64, No. 9, pp. 3820-3830, 2016.

[11] S. Zhang, J. Hiong, and S. He, "MIMO antenna system of two closely positioned PIFAs with high isolation", Electronics Letter, Vol. 45, pp. 771-773, 2009.

[12] Y. Cui, R. Li, and H. Fu, "A Broadband DualPolarized Planar Antenna for 2G/3G/LTE Base
Stations", IEEE Transactions on Antennas and Propagation, Vol. 62, No. 9, pp. 4836-4840, 2014.

[13] K. Arya, N. V. Anh, R. S. Aziz, B. Y. Park, and S. Park, "Dual Polarized Dual Antennas for 1.72.1 GHz LTE Base Stations", IEEE Antennas and Wireless Propagation Letters, Vol. 14, pp. 14271430, 2015.

[14] L. Song and J. Geng, "Analysis and Design of a Dual-polarized Printed Monopole Antenna", IETE Journal of Research, pp. 1-8, 2019.

[15] C. A. Balanis, Antenna Theory: Analysis and Design, John Wiley \& Sons, 2016.

[16] A. Chaudhari and R. K. Gupta, "A Simple TriBand MIMO Antenna Using a Single Ground Stub", Progress In Electromagnetics Research C, Vol. 86, pp. 191-201, 2018.

[17] Q. H. Kareem and M. J. Farhan, "Design a Linear and Circular Polarization MIMO Antennas Based on Compact Size Configurations with High Isolation and Stable Gain Characteristics for C- Band and WLAN / WiMAX Applications", International Journal of Intelligent Engineering and Systems, Vol. 14, No. 3, pp. 458-467, 2021.

[18] Y. Ban, C. Li, C. Sim, G. Wu, and K. Wong, "4G/5G Multiple Antennas for Future MultiMode Smartphone Applications", IEEE Access, Vol. 4, pp. 2981-2988, 2016.

[19] R. Mittra, Developments in Antenna Analysis and Design, Institute of Engineering and Technology, 2019.

[20] Y. Ding, Z. Du, K. Gong, and Z. Feng, "A novel dual-band printed diversity antenna for mobile terminals", IEEE Transaction Antennas Propagation, Vol. 55, No. 7, pp. 2088-2096, 2007.

[21] M. S. Sharawi, M. U. Khan, A. B. Numan, and D. N. Aloi, "A CSRR loaded MIMO antenna system for ISM band operation", IEEE Transactions on Antennas and Propagation, Vol. 61, No. 8, pp. 4265-4274, 2013.

[22] K. Wong, J. Lu, L. Chen, W. Li, and Y. Ban, "8antenna and 16-antenna arrays using the quadantenna linear array as a building block for the 3.5-GHz LTE MIMO operation in the smartphone", Microwave Optical Technology Letter, Vol. 58, pp. 174-181, 2016. 
[23] C. H. See, R. A. A. Alhameed, Z. Z. Abidin, N. J. Mcewan, and P. S. Excell, "Wideband printed MIMO/diversity monopole antenna for WiFi/WiMAX applications", IEEE Transactions on Antennas and Propagation, Vol. 60, No. 4, pp. 2028-2035, 2012.

[24] G. Das, A. Sharma, and R. K. Gangwar, "Dual port aperture coupled MIMO cylindrical dielectric resonator antenna with high isolation for WiMAX application", International Journal of $R F$ and Microwave Computer-Aided Engineering, Vol. 27, No. 7, e21107, 2017.

[25] C. Mao and Q. Chu, "Compact Co-radiator UWB-MIMO Antenna with Dual Polarization", IEEE Transactions on Antennas and Propagation, vol. 62, No. 9, pp. 4474-4480, 2014. 